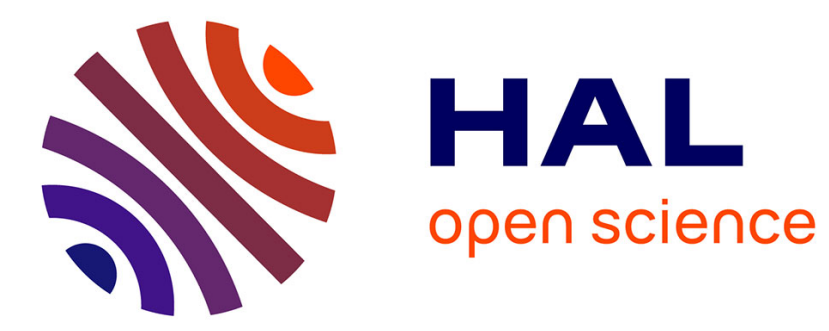

\title{
A spinorial characterization of hyperspheres
}

Oussama Hijazi, Sebastián Montiel

\section{To cite this version:}

Oussama Hijazi, Sebastián Montiel. A spinorial characterization of hyperspheres. Calculus of Variations and Partial Differential Equations, 2013, 48, pp.527-544. 10.1007/s00526-012-0560-x . hal01270168

\section{HAL Id: hal-01270168 \\ https://hal.science/hal-01270168}

Submitted on 8 Feb 2016

HAL is a multi-disciplinary open access archive for the deposit and dissemination of scientific research documents, whether they are published or not. The documents may come from teaching and research institutions in France or abroad, or from public or private research centers.
L'archive ouverte pluridisciplinaire HAL, est destinée au dépôt et à la diffusion de documents scientifiques de niveau recherche, publiés ou non, émanant des établissements d'enseignement et de recherche français ou étrangers, des laboratoires publics ou privés. 


\title{
A SPINORIAL CHARACTERIZATION OF HYPERSPHERES
}

\author{
OUSSAMA HIJAZI AND SEBASTIÁN MONTIEL
}

\begin{abstract}
Let $M$ be a compact orientable $n$-dimensional hypersurface, with nowhere vanishing mean curvature $H$, immersed in a Riemannian spin manifold $\bar{M}$ admitting a non trivial parallel spinor field. Then the first eigenvalue $\lambda_{1}\left(D_{M}^{H}\right)$ (with the lowest absolute value) of the Dirac operator $D_{M}^{H}$ corresponding to the conformal metric $\langle,\rangle^{H}=H^{2}\langle$,$\rangle , where \langle$,$\rangle is the induced met-$ ric on $M$, satisfies $\left|\lambda_{1}\left(D_{M}^{H}\right)\right| \leq \frac{n}{2}$. By applying the BourguignonGauduchon first variational formula, we obtain a necessary condition for $\left|\lambda_{1}\left(D_{M}^{H}\right)\right|=\frac{n}{2}$. As a consequence, we prove that round hyperspheres are the only hypersurfaces of the Euclidean space satisfying the equality in the Bär inequality
\end{abstract}

$$
\lambda_{1}\left(D_{M}\right)^{2} \leq \frac{n^{2}}{4 \operatorname{vol}(M)} \int_{M} H^{2} d V
$$

where $D_{M}$ stands now for the Dirac operator of the induced metric.

Dédié à Jean Pierre Bourguignon en témoignage de notre reconnaissance et amitié.

\section{IntRoduction AND SETTING OF THE PROBLEM}

Since the seventies intensive research has been done on the spectral properties of submanifolds of Riemannian manifolds. These spectral properties are usually referred to the scalar Laplace operator associated with the metric on the submanifold induced from the Riemannian structure of the ambient space. They mainly deal with two classes of problems. First, to get information on the spectrum of the Laplacian of a compact submanifold $M$ immersed in a given Riemannian ambient space $\bar{M}$, which is almost always a Riemannian manifold with a well-behaved curvature: the Euclidean space, the sphere, the projective space and other symmetric spaces. One looks for information given in terms of the extrinsic geometry of the submanifold, that is, in terms of

1991 Mathematics Subject Classification. Differential Geometry, Global Analysis, 53C27, 53C40, 53C80, 58G25.

Key words and phrases. Hypersurfaces, Mean Curvature, Dirac Operator, Eigenvalues.

The second author was partially supported by a Spanish MEC-FEDER grant No. MTM2007-61775. 
its second fundamental form, its mean curvature, etc. From a physical point of view, one tries to know the relation between the pure sounds given off by the submanifold and the way in which it is immersed into the ambient space ( see $[\mathrm{K}, \mathrm{GWW}]$ ). Secondly, to characterize some distinguished submanifolds (with a more symmetric geometry or second fundamental form) by a limiting behaviour of their eigenvalues, their multiplicities or their eigenfunctions.

To be precise, we shall recall one example of such results which is significant for the problem we shall consider in this paper. It was proved by Bleecker and Weiner in 1977 and, under a different form, by Reilly in $[\mathrm{Re}]$ and by Chen in [Ch]. Even though a suitable version remains to be true for arbitrary codimension, for the sake of simplicity we shall only refer to the case of hypersurfaces:

Theorem 1 ([BlW, Ch, Re]). The first non-zero eigenvalue of the Laplacian $\Delta$ acting on functions, of a compact hypersurface $M$ immersed into the Euclidean space $\mathbb{R}^{n+1}$, satisfies the following inequality

$$
\lambda_{1}(\Delta) \leq \frac{n}{\operatorname{vol}(M)} \int_{M} H^{2} d V
$$

where $\operatorname{vol}(M)$ is the volume of the submanifold and $H$ denotes its mean curvature function. Equality occurs if and only if the hypersurface is a (round) hypersphere.

This theorem can be obtained as an application of the following straightforward consequence of the Rayleigh variational characterization of $\lambda_{1}(\Delta)$ :

$$
\int_{M}(\Delta f)^{2} d V-\lambda_{1}(\Delta) \int_{M}|\nabla f|^{2} d V \geq 0
$$

inequality which is valid for each smooth function $f$ (not necessarily orthogonal to constants) on any compact Riemannian manifold $M$, and where equality holds only when, up to a constant, $f$ is an eigenfunction associated with $\lambda_{1}(\Delta)$, that is, only when

$$
\Delta f=-\lambda_{1}(\Delta)(f-c)
$$

for a certain $c \in \mathbb{R}$. In fact, take as a test function $f$ in the previous inequality the position vector function $F: M \rightarrow \mathbb{R}^{n+1}$ of the immersion of the hypersurface $M$ into $\mathbb{R}^{n+1}$, which is a vector valued function, and recall that, in this case,

$$
\Delta F=n H N \quad \text { and } \quad|\nabla F|^{2}=n,
$$

where $N$ is a unit vector field normal to $M$ and $H$ the mean curvature function (associated with this choice of $N$ ). 
From the above, if equality holds for a given immersion, then

$$
n H N=\Delta F=-\lambda_{1}(\Delta)\left(F-F_{0}\right)
$$

where $F_{0} \in \mathbb{R}^{n+1}$ is the center of mass of the hypersurface. Since $\lambda_{1}(\Delta)$ is non-zero, we deduce that the point $F_{0}$ lies on all the normal lines of $M$. As $M$ is compact, then $M$ is a hypersphere.

In the last years, some geometers of Submanifold Theory have been led to study diverse Dirac operators which naturally arise from the extrinsic geometry of surfaces, and some Riemannian geometers specialized in Spin Geometry have started to consider particular features of the Dirac operator on submanifolds (see [Am1, Am2, AF, Bä3, BFLPP, HMZ1, HMZ2, HMZ3, FLPP, T] and references therein). Their goal is just to examine the relation between the spectral properties of the classical Dirac operator and the geometry of submanifolds, particularly hypersurfaces, in the sense of the previous quoted results for the Laplacian.

In this direction, suppose that the manifold $M$ is now an orientable hypersurface immersed in an $(n+1)$-dimensional Riemannian manifold $\bar{M}$ which is spin (and this is the case for the most common ambient spaces in Submanifold Theory). Then it follows that $M$ inherits an induced spin structure and so we shall have also on $M$ the spinor bundle $\mathbb{S} M$ and the Dirac operator $D_{M}$ (see [BFGK, BHMM, LM]). This is the case, for example, when the ambient space $\bar{M}$ is $\mathbb{R}^{n+1}$. Moreover, the spin structure of $\mathbb{R}^{n+1}$ is very special. Indeed, Euclidean spaces, Calabi-Yau manifolds, hyper-Kähler manifolds, some other 8and 7-dimensional special Riemannian manifolds, and their Riemannian products are the only simply connected examples of Riemannian spin manifolds admitting non-trivial parallel spinor fields (see [Wa1]).

Let $\psi$ be such a parallel spinor field on $\mathbb{R}^{n+1}$, that we can choose with unit constant length. Denote by the same symbol its restriction to the hypersurface $M$ which is a section of $\mathbb{S} M$. In a way similar to that of Bleecker and Weiner, Bär used this spinor field $\psi$ as a test spinor in the variational characterization (Rayleigh quotient) of the lowest eigenvalues of the Dirac operator $D_{M}$ of $M$, namely

$$
\int_{M}\left|D_{M} \varphi\right|^{2} d V-\lambda_{1}\left(D_{M}\right)^{2} \int_{M}|\varphi|^{2} d V \geq 0
$$

where $\lambda_{1}\left(D_{M}\right)$ is the eigenvalue of $D_{M}$ with the lowest absolute value and $\varphi \in \Gamma(\mathbb{S} M)$ is any spinor field on $M$. From this inequality, since

$$
D_{M} \psi= \pm \frac{n}{2} H \psi \quad \text { and } \quad|\psi|^{2}=1
$$


we obtain the Bär inequality which can be seen as the analogue for the Dirac operator to the inequality for the scalar Laplacian obtained by Bleecker and Weiner (see [Gi, Chapter 5]).

Theorem 2 ([Bä3]). Let $M$ be a compact orientable hypersurface immersed into the Euclidean space $\mathbb{R}^{n+1}$ and let $\lambda_{1}\left(D_{M}\right)$ be the first eigenvalue (with the least absolute value) of the induced Dirac operator $D_{M}$ of $M$. Then

$$
\lambda_{1}\left(D_{M}\right)^{2} \leq \frac{n^{2}}{4 \operatorname{vol}(M)} \int_{M} H^{2} d V
$$

As we have seen, in the case of the Laplacian, Bleecker and Weiner had no difficulty to show that the equality is achieved only by round spheres. Yet in the case of the Dirac operator, it is an open problem to know when equality holds in the Bär inequality. Indeed, equality immediately implies that the test spinor field $\psi$ must be an eigenspinor for $D_{M}^{2}$. It is easy to see that this fact forces the mean curvature $H$ to be constant.

But this is not enough to get further information, except in the case where the hypersurface $M$ is embedded, that is, when we can ensure that it has no self-intersections. In this case, we can apply the wellknown Alexandrov theorem (proved in 1962, see [Al]): Any compact hypersurface embedded in the Euclidean space with constant mean curvature must be a hypersphere. Because of this result, during the sixties and seventies of the last century, most of the geometers of submanifolds thought that perhaps the spheres were the only hypersurfaces immersed into the Euclidean space with constant mean curvature (see [Ho]). But since 1983, many examples of this class of hypersurfaces which are not round spheres, have been constructed by Hsiang, Ten and Yu [HTY], when the dimension of the ambient space is even, and by Wente [We] and Kapouleas [Ka1, Ka2] in the three-dimensional space (in this case, necessarily with genus greater than one, see again [Ho]).

In short, if equality holds in Inequality (1), the mean curvature $H$ of the hypersurface $M$ is constant and so, as pointed out before, the restriction of the parallel spinor field $\psi$ is an eigenspinor of the Dirac operator $D_{M}$ corresponding to one of the eigenvalues $\pm \frac{n}{2} H$ and this eigenvalue must be the eigenvalue of $D_{M}$ with the least absolute value. So an interesting question is: As for the scalar Laplacian, is it true that only hyperspheres satisfy equality in the Bär Inequality (1), even in the general immersed case?

As we have just observed, since the problem of determining the hypersurfaces of the Euclidean space attaining the equality in Inequality 
(1) is equivalent to the problem of characterizing constant mean curvature hypersurfaces of $\mathbb{R}^{n+1}$ satisfying $\left|\lambda_{1}\left(D_{M}\right)\right|=\frac{n}{2}|H|$, the question considered in this paper can be viewed as the spinorial version of a well-known problem posed by Yau and Ogiue for the scalar Laplacian on minimal hypersurfaces of the sphere (see $[\mathrm{Y}, \mathrm{O}]$ ): the determination of the so-called minimal surfaces of the sphere immersed by first eigenfunctions, that is, those minimal hypersurfaces of the sphere for which their dimension (which is always an eigenvalue of the scalar Laplacian) is exactly the first non-zero eigenvalue of the Laplacian. This is why we will also refer to the hypersurfaces attaining the equality in (1), as constant mean curvature hypersurfaces immersed by first eigenspinors.

We shall give some answers to these questions. Indeed, first we shall prove the following theorem:

Theorem 3. Let $M$ be a compact hypersurface immersed in the Euclidean space $\mathbb{R}^{n+1}$ with constant mean curvature $H$. It is known (see [Bä3, BFGK, Bu]) that both real numbers $\pm \frac{n}{2} H$ are eigenvalues of the induced Dirac operator $D_{M}$. If the multiplicity mult $\left( \pm \frac{n}{2} H\right)$ of any of them satisfies

$$
\text { mult }_{D_{M}}\left( \pm \frac{n}{2} H\right)<2^{\left[\frac{n}{2}\right]+1},
$$

then we have exactly

$$
\text { mult }_{D_{M}}\left( \pm \frac{n}{2} H\right)=2^{\left[\frac{n}{2}\right]}
$$

for both of them and $M$ must be a round hypersphere.

As a consequence, we shall deduce our main result:

Theorem 4. Under the conditions of Theorem 2, equality holds in the Bär Inequality (1) if and only if $M$ is a round hypersphere. That is, the only compact constant mean curvature hypersurfaces of the Euclidean space immersed by first eigenspinors are hyperspheres.

\section{Preliminaries on SPIN MANifoldS}

Let $(\bar{M},\langle\rangle$,$) be an (n+1)$-dimensional Riemannian spin manifold and denote by $\nabla$ the Levi-Civita connection on its tangent bundle $T \bar{M}$. We choose a spin structure on $\bar{M}$ and consider the corresponding spinor bundle $\mathbb{S} \bar{M}$, a rank $2^{\left[\frac{n+1}{2}\right]}$ complex vector bundle which depends not only on the chosen spin structure but also on the Riemannian metric on $\bar{M}$. Denote by $\gamma$ the Clifford multiplication

$$
\gamma: \mathbb{C} \ell(\bar{M}) \longrightarrow \operatorname{End}(\mathbb{S} \bar{M})
$$


which is a fibre preserving algebra morphism. Then $\mathbb{S} \bar{M}$ becomes a bundle of complex left modules over the Clifford bundle $\mathbb{C} \ell(\bar{M})$. When $(n+1)$ is even, the spinor bundle splits into

$$
\mathbb{S} \bar{M}=\mathbb{S} \bar{M}^{+} \oplus \mathbb{S} \bar{M}^{-}
$$

where $\mathbb{S} \bar{M}^{ \pm}$are the \pm 1-eigenspaces of the endomorphism $\gamma\left(\omega_{n+1}\right)$, with $\omega_{n+1}=i^{\left[\frac{n+2}{2}\right]} e_{1} \cdot e_{2} \cdots e_{n+1}$, the complex volume form.

On the spinor bundle $\mathbb{S} \bar{M}$, one has (see [LM]) a natural Hermitian metric, denoted as the Riemannian metric on $\bar{M}$ by $\langle$,$\rangle , and$ the spinorial Levi-Civita connection $\nabla$ acting on spinor fields. The Hermitian metric and the connection are compatible with the Clifford multiplication (2) and compatible with each other. That is

$$
\begin{gathered}
X\langle\psi, \varphi\rangle=\left\langle\nabla_{X} \psi, \varphi\right\rangle+\left\langle\psi, \nabla_{X} \varphi\right\rangle \\
\langle\gamma(X) \psi, \gamma(X) \varphi\rangle=|X|^{2}\langle\psi, \varphi\rangle \\
\nabla_{X}(\gamma(Y) \psi)=\gamma\left(\nabla_{X} Y\right) \psi+\gamma(Y) \nabla_{X} \psi,
\end{gathered}
$$

for any spinor fields $\psi, \varphi \in \Gamma(\mathbb{S} \bar{M})$ and any tangent vector fields $X, Y \in$ $\Gamma(T \bar{M})$. Since $\nabla \omega_{n+1}=0$, so when $(n+1)$ is even, the decomposition (3) becomes orthogonal and $\nabla$ preserves this decomposition.

The Dirac operator $D$ on $\mathbb{S} \bar{M}$ is the first order elliptic differential operator, locally given by

$$
D \varphi=\sum_{i=1}^{n+1} \gamma\left(e_{i}\right) \nabla_{e_{i}} \varphi,
$$

where $\left\{e_{1}, \ldots, e_{n+1}\right\}$ is a local orthonormal frame of $T \bar{M}$ and $\varphi \in$ $\Gamma(\mathbb{S} \bar{M})$ is any spinor field on $\bar{M}$. It is clear, from this definition, that the function $\langle D \varphi, \varphi\rangle$ is nothing but the trace of a symmetric two-tensor associated with any spinor field $\varphi$, the so-called energy-momentum tensor $T_{\varphi}$ which is defined as follows (see [BG, Hi2, FK])

$$
T_{\varphi}(X, Y)=\frac{1}{2}\left\langle\gamma(X) \nabla_{Y} \varphi+\gamma(Y) \nabla_{X} \varphi, \varphi\right\rangle
$$

for any $X, Y \in \Gamma(\mathbb{S} \bar{M})$. Also from the definition, we see that, when $(n+1)$ is even, the Dirac operator interchanges positive and negative spinor fields, that is,

$$
D: \Gamma\left(\mathbb{S} \bar{M}^{ \pm}\right) \longrightarrow \Gamma\left(\mathbb{S} \bar{M}^{\mp}\right) .
$$

Suppose now that the manifold $\bar{M}$ is compact. Then the ellipticity and the self-adjointness of the Dirac operator $D$ imply that this 
operator has a discrete real spectrum that we list in this way:

$-\infty \leftarrow \cdots \leq \lambda_{-k} \leq \cdots \leq \lambda_{-1} \leq 0 \leq \lambda_{1} \leq \cdots \leq \lambda_{k} \leq \cdots \rightarrow+\infty$,

with all these eigenvalues repeated according their multiplicities for all $k \in \mathbb{N}$. This spectrum depends on the spin structure and on the Riemannian metric chosen on the manifold.

\section{HypersurfaCES AND INDUCED STRUCTURES}

In this section, we compare the restriction $\$ M$ of the spinor bundle of the spin manifold $\bar{M}$ to an orientable hypersurface $M$ immersed into $\bar{M}$ and its Dirac-type operator $\not D$ to the intrinsic spinor bundle $\mathbb{S} M$ of the induced spin structure of $M$ and its Dirac operator $D_{M}$. These facts are well-known (see for example [Bu, Tr, Bä2, BFGK] or our previous papers [HMZ1, HMZ2, HMZ3, HMR1, HMR2, HM]). For completeness, we introduce the key facts.

Let $\nabla$ be the Levi-Civita connection associated with the induced Riemannian metric on $M$. The Gauß formula says that

$$
\nabla_{X} Y=\nabla_{X} Y-\langle A X, Y\rangle N
$$

where $X, Y$ are vector fields tangent to the hypersurface $M$, the vector field $N$ is a global unit field normal to $M$ and $A$ stands for the shape operator corresponding to $N$, that is,

$$
\nabla_{X} N=-A X, \quad \forall X \in \Gamma(T M) .
$$

We have that the restriction

$$
\mathbb{S} M:=\mathbb{S} \bar{M}_{\mid M}
$$

is a left module over $\mathbb{C} \ell(M)$ for the induced Clifford multiplication

$$
\not /: \mathbb{C} \ell(M) \longrightarrow \operatorname{End}(\$ M)
$$

given by

$$
\not(X) \psi=\gamma(X) \gamma(N) \psi
$$

for every $\psi \in \Gamma(\$ M)$ and $X \in \Gamma(T M)$. Consider on $\$ M$ the Hermitian metric $\langle$,$\rangle induced from that of \mathbb{S} \bar{M}$. This metric immediately satisfies the compatibility condition (5) if one puts on $M$ the Riemannian metric induced from $\bar{M}$ and the Clifford multiplication $\not$ defined in (10). Consider also the connection $\nabla$ on $\$ M$ given by

$$
\nabla_{X} \psi=\nabla_{X} \psi-\frac{1}{2} \gamma(A X) \gamma(N) \psi=\nabla_{X} \psi-\frac{1}{2} \chi(A X) \psi,
$$

for every $\psi \in \Gamma(\mathbb{S} M)$ and $X \in \Gamma(T M)$. Note that a suitable use of the Gauß formula (8) shows that the compability conditions (4) and (6) are also satisfied for $(\$ M, \not,\langle\rangle,, \nabla)$. 
Denote by $\not D: \Gamma(\mathbb{D} M) \rightarrow \Gamma(\mathbb{D} M)$ the Dirac operator associated with the Dirac bundle $\$ M$ over the hypersurface, which is defined by

$$
\not D \psi=\sum_{j=1}^{n} \not /\left(e_{j}\right) \nabla_{e_{j}} \psi
$$

where $\psi \in \Gamma(\$ M)$ and $\left\{e_{1}, \ldots, e_{n}\right\}$ is a local orthonormal frame of $T M$. Since, as we can see from (10) and (11), the connection $\nabla$ does not depend on the choice of the normal $N$ on $M$ while $\not /$ changes sign for the opposite orientation, hence the same is true for $\mathbb{D}$.

It is a well known fact that $\not D$ is a first order elliptic differential operator which is formally $L^{2}$-selfadjoint. By (11), for any spinor field $\psi \in \Gamma(\$ M)$, we have

$$
\not D \psi=\frac{n}{2} H \psi-\gamma(N) \sum_{j=1}^{n} \gamma\left(e_{j}\right) \nabla_{e_{j}} \psi,
$$

where $H=\frac{1}{n} \operatorname{trace} A$ is the mean curvature of $M$ corresponding to the orientation $N$. Note that if the spinor field $\psi$ is the restriction to the hypersurface $M$ of a spinor field on the ambient space $\bar{M}$, then both spinor fields in $\Gamma(\mathbb{S} \bar{M})$ and $\Gamma(\mathbb{S} M)$, will be denoted by the same symbol.

Lemma 5. For any spinor field $\psi \in \Gamma(\$ M)$ and any tangent vector field $X \in \Gamma(T M)$, the following relations hold

$$
\begin{aligned}
\nabla_{X}(\gamma(N) \psi) & =\gamma(N) \nabla_{X} \psi, \\
\not D(\gamma(N) \psi) & =-\gamma(N) \not D \psi .
\end{aligned}
$$

The proof is straightforward using (11) and (9). In particular, the second of these inequalities implies that, when $M$ is a compact manifold without boundary, the spectrum of $\not D$ must be symmetric with respect to zero. This does not occur necessarily for $D_{M}$. For the purpose of relating the spectra of $\not D$ and $D_{M}$ in a precise way, we gather in the following proposition, well-known results that we will need later.

Proposition 6. Let $\bar{M}$ be an $(n+1)$-dimensional Riemannian spin manifold and $(\mathbb{S} \bar{M}, \gamma)$ its spinor bundle, where $\gamma$ denotes the corresponding Clifford multiplication. Consider an orientable hypersurface $M$ immersed in $\bar{M}$ and let $(\mathbb{\$} M, \not)$ and $\left(\mathbb{S} M, \gamma_{M}\right)$ be respectively the induced Dirac bundle and the spinor bundle of the induced spin structure on $M$. Denote by $\not D$ and $D_{M}$ the corresponding Dirac operators.

a) When the dimension $n$ of $M$ is even we have $(\mathbb{D} M, \not, \not D) \equiv$ $\left(\mathbb{S} M, \gamma_{M}, D_{M}\right)$ and the decomposition $\mathbb{\$} M=\mathbb{\$} M^{+} \oplus \mathbb{\$} M^{-}$, given by $\mathbb{S} M^{ \pm}:=\{\eta \in \mathbb{S} M \mid i \gamma(N) \eta= \pm \eta\}$, corresponds, up to the above identification, to the chirality decomposition of the spinor 
bundle $\mathbb{S} M$. Hence $\not D$ interchanges $\mathbb{\$} M^{+}$and $\mathbb{\$} M^{-}$, as one can also easily see from Lemma 5.

b) When $n$ is odd, the chirality decomposition of $\mathbb{S} \bar{M}$ into positive and negative spinors induces an orthogonal and $\not$, DD-invariant decomposition $\mathbb{S} M=\mathbb{\$} M_{+} \oplus \mathbb{S} M_{-}$, with $\mathbb{\$} M_{ \pm}:=\left(\mathbb{S} \bar{M}^{ \pm}\right)_{\mid M}$, in such a way that $\left(\mathbb{\$} M_{ \pm}, \not, \not D_{\mid \mathbb{} M_{ \pm}}\right) \equiv\left(\mathbb{S} M, \pm \gamma_{M}, \pm D_{M}\right)$. Moreover, we have the following two isomorphisms: $\gamma(N): \mathbb{\$} M_{ \pm} \longmapsto$ $\$ M_{\mp}$.

Furthermore, if $M$ is compact without boundary, then the discrete spectra of the elliptic self-adjoint operators $\not D$ and $D_{M}$, satisfy the following:

c) Spec $\not D$ is symmetric with respect to zero.

d) If $\operatorname{dim} M$ is even, we have that $\operatorname{Spec} \not D=\operatorname{Spec} D_{M}$, and that $\operatorname{mult}_{\not D}(\lambda)=$ mult $_{D_{M}}(\lambda)$, for all $\lambda \in \operatorname{Spec} D_{M}$.

e) If $\operatorname{dim} M$ is odd, we have mult $\not_{\mid \ngtr M_{ \pm}}(\lambda)=$ mult $_{D_{M}}( \pm \lambda)$, and so $\operatorname{Spec} \not D=\operatorname{Spec} D_{M} \cup\left(-\operatorname{Spec} D_{M}\right)$. Moreover,

$\lambda \in \operatorname{Spec} D_{M}$ and $-\lambda \notin \operatorname{Spec} D_{M} \Longrightarrow \operatorname{mult}_{\not D}(\lambda)=\operatorname{mult}_{D_{M}}(\lambda)$, $\pm \lambda \in \operatorname{Spec}_{M} \Longrightarrow \operatorname{mult}_{\not D}(\lambda)=\operatorname{mult}_{D_{M}}(\lambda)+\operatorname{mult}_{D_{M}}(-\lambda)$.

Remark 1. Although, by definition, sections of the restricted bundle $\$ M$ are defined only on the hypersurface $M$ and not on the whole space $\bar{M}$, we may consider derivatives of the form $\nabla_{X} \psi$, where $\nabla$ is the spinorial Levi-Civita connection on the ambient space and where $\psi \in$ $\Gamma(\$ M)$, provided that the field $X$ is tangent to $M$. In fact, at any point of $M$, the spinor field $\psi$ can be locally extended to $\bar{M}$, we compute the covariant derivative of the extension and restrict again to $M$. The result does not depend on the chosen local extension. Alternatively, if we think of the restricted bundle $\mathbb{S} M$ as the spinor bundle $\mathbb{S} M$, according to the above identifications, via the Gauß formula (11), we may define the connection $\nabla$ in terms of the spinorial Levi-Civita connection $\nabla$, the Clifford multiplication $\not y$ on $\mathbb{S} M$ and the shape operator $A$ of the hypersurface.

The above considerations regarding the relation between $\not D$ and $D_{M}$ and the nature of the assertions that we want to prove in Theorems 3 and 4 , allow us to work henceforth only with $\$ M$ and $\not D$, and shall refer to them as the spinor bundle and the Dirac operator of the induced spin structure of the hypersurface $M$. From Remark 1 and (12), we deduce the following well-known result:

Proposition 7 ([Bä3, BFGK, Bu]). Let $M$ be an orientable hypersurface immersed into a Riemannian spin manifold $\bar{M}$ admitting a parallel 
spinor field $\psi \in \Gamma(\mathbb{S} \bar{M})$. Then the restriction $\psi \in \Gamma(\mathbb{\$} M)$ satisfies

$$
\not D \psi=\frac{n}{2} H \psi \text {. }
$$

If $H$ is constant, then $\frac{n}{2} H$ belongs to the spectrum of $\not D$, hence $\lambda_{1}(\not D) \leq$ $\frac{n}{2}|H|$. If $\lambda_{1}(\not D)=\frac{n}{2}|H|$, we shall say that $M$ is immersed in $\bar{M}$ by first eigenspinors.

Remark 2. Consider the case of the round unit hypersphere $M=\mathbb{S}^{n}$ as an embedded hypersurface of $\mathbb{R}^{n+1}$, an ambient space which admits a maximal number of independent parallel spinor fields. It is wellknown that the eigenvalues of the Dirac operator $\not D$ of the unique spin structure on $\mathbb{S}^{n}$ with the lowest absolute value are $\pm \frac{n}{2}$ (see $[\mathrm{Gi}$, Theorem 2.1.3]), both with multiplicity $2^{\left[\frac{n+1}{2}\right]}$. Moreover it is also clear that $H= \pm 1$ according to the choice of the orientation, hence in this case $\lambda_{1}(\not D)=\frac{n}{2}|H|$.

\section{Conformal Change of the hypersurface metric}

Suppose now that the mean curvature $H$ of the immersed (connected) hypersurface $M$ of the spin manifold $\bar{M}$ has no zeros. Fix the orientation and the corresponding unit normal field so that $H>0$. Consider on $M$ the conformal metric $\langle,\rangle^{H}=H^{2}\langle$,$\rangle . If we denote$ by $\mathbb{S}^{H} M$ the spin bundle corresponding to this conformal metric and the same spin structure. We shall identify the two spinor bundles $\$ M$ and $\$^{H} M$ using the isomorphism given in [Hi1]. Due to the existence of this isometric identification, from now on, we shall denote the two spinor bundles by the same symbol $\$ M$. With this identification, the corresponding Clifford multiplications and spin connections are related as follows:

$$
\not^{H}=H \not, \quad \nabla_{X}^{H}-\nabla_{X}=-\frac{1}{2 H} \not(X) \not(\nabla H)+\langle X, \nabla H\rangle,
$$

for all $X \in \Gamma(T M)$. From this, we can easily find the following relation between the two Dirac operators $\not D$ and $\not D^{H}$ on $\$ M$ relative to the two conformal metrics (see [Hit, Hi1]):

$$
\not D^{H}\left(H^{-\frac{n-1}{2}} \varphi\right)=H^{-\frac{n+1}{2}} \not D \varphi, \quad \forall \varphi \in \Gamma(\mathbb{\$} M) .
$$

Remark 3. When the mean curvature $H$ is constant, the metrics $\langle,\rangle^{H}$ and $\langle$,$\rangle are homothetic. In this case the conformal covariance (14)$ becomes the following change of scale: $\not D^{H}=\frac{1}{H} \not D$. 
Suppose now that there exists a non-trivial parallel spinor field $\psi \in$ $\Gamma(\mathbb{S} \bar{M})$ and take as $\varphi$ in (14) its restriction $\psi \in \Gamma(\mathbb{S} M)$. Using Proposition 7 , we obtain

$$
\not D^{H}\left(H^{-\frac{n-1}{2}} \psi\right)=H^{-\frac{n+1}{2}} \not D \psi=\frac{n}{2} H^{-\frac{n-1}{2}} \psi .
$$

From this equality we can deduce the following result.

Proposition 8. Let $M$ be an orientable hypersurface immersed into a Riemannian spin manifold $\bar{M}$ admitting a parallel spinor field $\psi \in$ $\Gamma(\mathbb{S} \bar{M})$ and suppose that the mean curvature of $M$, after a suitable choice of the unit normal, satisfies $H>0$. Denote by $\psi^{H}$ the spinor field $H^{-\frac{n-1}{2}} \psi \in \Gamma(\$ M)$. Then we have $\not^{H} \psi^{H}=\frac{n}{2} \psi^{H}$. As a consequence, $\frac{n}{2}$ belongs to the spectrum of $\not D^{H}$, hence

$$
\lambda_{1}\left(\not D^{H}\right) \leq \frac{n}{2}
$$

Remark 4. It is important to note that, when the mean curvature $H$ of the hypersurface is constant and $\lambda_{1}(\not D)=\frac{n}{2} H$, that is, when $M$ is immersed by first eigenspinors (see Proposition 7), then from Remark 3 , we get $\lambda_{1}\left(\not D^{H}\right)=\frac{n}{2}$. That is, in this case, the equality is achieved in (15).

Remark 5. Proposition 8 can be stated in terms of a spectral functional $\mathcal{F}_{1}$ defined on the space of immersions $\iota: M \rightarrow \bar{M}$ of a compact $n$-dimensional manifold $M$ into a Riemannian spin $(n+1)$-dimensional manifold $(\bar{M},\langle\rangle$,$) with non vanishing mean curvature, that we shall$ denote by $\operatorname{Imm}^{+}(M, \bar{M})$. This functional is defined by

$$
\mathcal{F}_{1}: \iota \in \operatorname{Imm}^{+}(M, \bar{M}) \longmapsto \lambda_{1}\left(\not D^{H_{\iota}}\right) \in \mathbb{R},
$$

where $\lambda_{1}\left(\not D^{H_{\iota}}\right)$ is the first non-negative eigenvalue of the Dirac operator $\not D^{H_{\iota}}$ of the induced spin structure corresponding to the conformal metric on $M$ given by

$$
\langle,\rangle^{H_{\iota}}=H_{\iota}^{2} \iota^{*}\langle,\rangle .
$$

This functional $\mathcal{F}_{1}$ is clearly invariant by homotheties of the ambient space metric. Proposition 8 can be paraphrased by saying that this functional is bounded from above by $\frac{n}{2}$ provided that there exists a non-trivial parallel spinor field on the ambient space $\bar{M}$. In fact, when $\bar{M}=\mathbb{R}^{n+1}$, from Remark 2 and Proposition 8, we have that this bound is achieved by hyperspheres of arbitrary radius, so $\frac{n}{2}$ is a maximum. In general, from Remark 4, we can see that all constant mean curvature hypersurfaces in Riemannian spin manifolds with non-trivial parallel spinors immersed by first eigenspinors attain this maximum, too. In the 
following section, we shall see that, in the Euclidean ambient space, the unique hypersurfaces achieving this maximum are the hyperspheres.

\section{Hypersurfaces having $\frac{n}{2}$ AS a CRItical eigenvalue of $\not D^{H}$}

Suppose that $\iota: M \rightarrow \bar{M}$ is an immersion of a compact orientable $n$-dimensional manifold $M$, into a Riemannian spin manifold $\bar{M}$, whose mean curvature function $H$ is positive and such that $\lambda_{k}\left(\not D^{H}\right)=\frac{n}{2}$, for some $k \in \mathbb{N}$. Note that here we are not assuming the existence of a non trivial parallel spinor field on $\bar{M}$. Consider an analytic variation $\iota(t)$ of the immersion $\iota$, that is, a family of immersions analytically indexed by $t \in]-\epsilon, \epsilon[$ such that $\iota(0)=\iota$. Since they are homotopic, all the immersions $\iota(t)$ of the variation induce on $M$ the same spin structure from the spin structure on $\bar{M}$. Moreover, the variation induces an analytic deformation of the Riemannian metric $\langle,\rangle^{H}$ on $M$, namely,

$$
\langle,\rangle^{H}(t)=\langle,\rangle(t)^{H(t)}=H(t)^{2}\langle,\rangle(t),
$$

where $H(t)$ (which remains to be positive if $\epsilon$ is small enough) and $\langle\rangle,(t)$ are respectively the mean curvature and the induced metric of the immersion $\iota(t)$. This deformation of the metric determines an analytic family of Dirac operators $\not D^{H}(t)$ given by $\not D^{H}(t)=\not D(t)^{H(t)}$, each acting on the different spinor bundles $\mathbb{\$}_{t}^{H(t)} M$ corresponding to the common induced spin structure and the metrics $\langle,\rangle^{H}(t)$, where we are using the notation introduced in Section 4 for conformal changes of the metric and where the spinor bundle $\mathbb{S}_{t} M$ corresponds to the induced spin structure and to the metric $\langle\rangle,(t)$. In order to compare the different deformed Dirac operators $\not D^{H}(t)$ with the Dirac operator $\not D^{H}(0)=\not D^{H}$ associated to the original immersion, it is necessary to

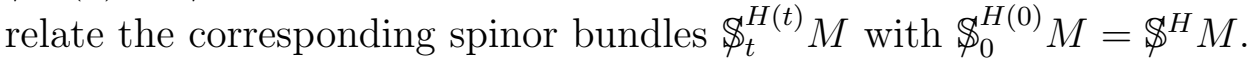
There is no canonical way to do this, but Bourguignon and Gauduchon determined in $[\mathrm{BG}]$ a specific way to define an isometry between two spinor bundles corresponding to two different metrics and to a same spin structure on a given manifold. This isometry allowed them to consider the two associated Dirac operators as two operators acting on the same bundle and to obtain an explicit relation between them ([BG, Théorème 20]). It is also important to observe that, when the two metrics are conformally related, the Bourguignon and Gauduchon identification coincides with that of Section 4. Then, using this isometry, we may see the one-parameter family $\not D^{H}(t)$ as an analytic family of Dirac operators acting on the same spinor bundle $\mathbb{S}^{H} M \cong \mathbb{\$} M$.

Now, we are interested in the first variation of the eigenvalues $\lambda_{k}\left(\not D^{H}\right)$ with respect to the analytic deformation $\iota(t)$ of the immersion $\iota$, that 
is, with respect to the variation of the metric $\langle,\rangle^{H}(t)$ on $M$. In other words, we want to compute the derivatives

$$
\left.\frac{d}{d t}\right|_{t=0} \mathcal{F}_{k}(t)=\left.\frac{d}{d t}\right|_{t=0} \lambda_{k}(t)
$$

provided that they exist, where

$$
\mathcal{F}_{k}(t)=\mathcal{F}_{k}(\iota(t)), \quad \lambda_{k}(t)=\lambda_{k}\left(\not D^{H}(t)\right) .
$$

For this, one can see in $[\mathrm{BG}]$ that the Rellich-Kato theory of unbounded self-adjoint operators can be applied to the analytic family $\not D^{H}(t)$ acting on $\$ M$. Denote by $m$ the dimension of the eigenspace $E_{k}\left(\not D^{H}\right)$ associated with the eigenvalue $\lambda_{k}\left(\not D^{H}\right)$. Then, for each $t \in$ ] $-\epsilon, \epsilon\left[\right.$, there exist $m$ eigenvalues $\Lambda_{k, 1}(t), \ldots, \Lambda_{k, m}(t)$ of $\not D^{H}(t)$ associated with an $L^{2}\left(M,\langle,\rangle^{H}(t)\right)$-orthonormal family of eigenspinors $\psi_{k, 1}(t), \ldots, \psi_{k, m}(t) \in \Gamma(\mathbb{S} M)$ of $\not D^{H}(t)$, both $\Lambda_{k, i}(t)$ and $\psi_{k, i}(t)$ depending analytically on $t$, for $i=1, \ldots, m$, such that $\Lambda_{k, 1}(0)=\cdots=$ $\Lambda_{k, m}(0)=\lambda_{k}\left(\not D^{H}\right)$ and $\not D^{H}(t) \psi_{k, i}(t)=\Lambda_{k, i}(t) \psi_{k, i}(t)$, for all $\left.t \in\right]-\epsilon, \epsilon[$, and $i=1, \ldots, m$.

Then, in order to study the critical points of $\mathcal{F}_{k}$, we need to know the derivatives $\Lambda_{k, i}^{\prime}(0)$ of the $m$ branches $\Lambda_{k, i}(t)$ passing through $\lambda_{k}\left(\not D^{H}\right)$ at $t=0$. The values of these derivatives were computed in $[\mathrm{BG}$, Théorème 24]: for each $i=1, \ldots, m$,

$$
\left.\frac{d}{d t}\right|_{t=0} \mathcal{F}_{k}^{(i)}(t)=\left.\frac{d}{d t}\right|_{t=0} \Lambda_{k, i}(t)=-\frac{1}{2} \int_{M}\left\langle T_{\psi_{i}}^{H}, h\right\rangle^{H} d V^{H},
$$

where $d V^{H}$ is the Riemannian density of the metric $\langle,\rangle^{H}$ on $M$ and $\psi_{i}=\psi_{k, i}(0)$ is a fortiori an $L^{2}\left(M,\langle,\rangle^{H}\right)$-orthonormal basis of the eigenspace $E_{k}\left(\not D^{H}\right)$. Here $T_{\psi}^{H}$ stands for the energy-momentum tensor (as defined in $(7)$ ) of the spinor $\psi \in \Gamma(\mathbb{\$} M)$, calculated with respect to the conformal metric $\langle,\rangle^{H}$ on $M$, that is, for all vector fields $X, Y$ tangent to $M$ :

$$
T_{\psi}^{H}(X, Y)=\frac{1}{2}\left\langle\not^{H}(X) \nabla_{Y}^{H} \psi+\not^{H}(Y) \nabla_{X}^{H} \psi, \psi\right\rangle,
$$

and $h$ is the variational field associated with the metric variation $\langle,\rangle^{H}(t)$, that is,

$$
h=\left.\frac{d}{d t}\right|_{t=0}\langle,\rangle^{H}(t)
$$


It can be shown (see $[\mathrm{BG}])$ that these $m$ derivatives $\left.\frac{d}{d t}\right|_{t=0} \mathcal{F}_{k}^{(i)}(t)$ are just the eigenvalues of the quadratic form $Q_{h}$ defined on the $m$ dimensional eigenspace $E_{k}\left(\not D^{H}\right)$ as follows:

$$
\varphi \in E_{k}\left(\not D^{H}\right) \longmapsto Q_{h}(\varphi)=-\frac{1}{2} \int_{M}\left\langle T_{\varphi}^{H}, h\right\rangle^{H} d V^{H} .
$$

Our next goal is to compute $Q_{h}(\varphi)$ in terms of the metric $\langle$, induced on $M$. First note that since the functional $\mathcal{F}_{k}$ is geometric, it is invariant under reparametrizations of $M$, so there is no loss of generality by assuming that the variation $\iota(t)$ of the immersion $\iota$ is a normal variation, that is,

$$
\left.\frac{d}{d t}\right|_{t=0} \iota(t)=f N
$$

where $f$ is a smooth function defined on $M$.

Then, using (16) and (18), the variational tensor field $h$ corresponding to the metric deformation $\langle,\rangle^{H}(t)$ is precisely

$$
h=2 H\left(\left.\frac{d}{d t}\right|_{t=0} H(t)\right)\langle,\rangle+\left.H^{2} \frac{d}{d t}\right|_{t=0}\langle,\rangle(t) .
$$

Now, it is a well-known fact in Submanifold Theory that the variation of the induced metric associated with the normal variation (20) of the immersion is given by

$$
\left.\frac{d}{d t}\right|_{t=0}\langle,\rangle(t)=-2 f\langle A,\rangle,
$$

where $A$ is the shape operator, and that the corresponding variation of the mean curvature is given by

$$
\left.\frac{d}{d t}\right|_{t=0} H(t)=\frac{1}{n}\left(\Delta f+|A|^{2} f+\overline{\operatorname{Ric}}(N, N) f\right),
$$

with $\overline{\mathrm{Ric}}$, the Ricci tensor of the manifold $\bar{M}$ (see [Mo], for instance). So, finally, we obtain

$$
h=\frac{2}{n} H\left(\Delta f+|A|^{2} f+\overline{\operatorname{Ric}}(N, N) f\right)\langle,\rangle-2 H^{2} f\langle A,\rangle .
$$

We also need to compute the energy-momentum tensor $T_{\varphi}^{H}$ associated with an eigenspinor $\varphi \in E_{k}\left(\not D^{H}\right)$. By definition of this tensor (see (7) and (17)) and the relation between the Levi-Civita connections and the Clifford multiplications of two conformally related metrics established in (13), we get the following simple covariance rule [Hi2]

$$
T_{\varphi}^{H}=H T_{\varphi}
$$


Choose any eigenspinor $\varphi \in E_{k}\left(\not D^{H}\right)$. Since $H>0$, we have that

$$
\varphi=\psi^{H}=H^{-\frac{n-1}{2}} \psi
$$

for a unique $\psi \in \Gamma(\$ M)$. Then by the conformal covariance (14) and the fact that $\varphi$ is an eigenspinor of $\not D^{H}$, we get

$$
\lambda_{k}\left(\not D^{H}\right) \varphi=\not D^{H} \varphi=H^{-\frac{n+1}{2}} \not D \psi,
$$

hence

$$
\not D \psi=\lambda_{k}\left(\not{ }^{H}\right) H \psi=\frac{n}{2} H \psi .
$$

Similarly, the conformal covariance (22) of the energy-momentum tensor can be expressed in terms of the spinor field $\psi$. It suffices to use the properties of $\psi$ and the conformal covariance

$$
T_{g \varphi}=g^{2} T_{\varphi}, \quad \forall g \in C^{\infty}(M)
$$

which can be deduced from the definition of the tensor $T_{\varphi}$ (see (7)). So, we have

$$
T_{\varphi}^{H}=H T_{H^{-\frac{n-1}{2}} \psi}=H^{-n+2} T_{\psi} .
$$

Finally we are in position to compute the integrand in the quadratic form (19) which controls the first derivative of the functional $\mathcal{F}_{k}$ at $t=0$. First, we observe that

$$
\left\langle T_{\varphi}^{H}, h\right\rangle^{H}=\frac{1}{H^{4}}\left\langle T_{\varphi}^{H}, h\right\rangle,
$$

since this a scalar product of two-tensors. Now, using (21), (23), (24) and (25), we deduce the expression

$$
\left\langle T_{\varphi}^{H}, h\right\rangle^{H}=\frac{1}{H^{n}}\left(\left(\Delta f+|A|^{2} f+\overline{\operatorname{Ric}}(N, N) f\right)|\psi|^{2}-2 f\left\langle A, T_{\psi}\right\rangle\right)
$$

In order to compute the contraction $\left\langle A, T_{\psi}\right\rangle$, take the squared length in the first equality of (11), where the two connections $\nabla$ and $\nabla$ on the restricted bundle $\$ M$ are related, considering that, for all $X, Y \in$ $\Gamma(T M)$, the corresponding version of (7) is now,

$$
T_{\psi}(X, Y)=\frac{1}{2}\left\langle\not(X) \nabla_{Y} \psi+\not(Y) \nabla_{X} \psi, \psi\right\rangle .
$$

Hence we get

$$
\left\langle A, T_{\psi}\right\rangle=-|\nabla \psi|^{2}+|\nabla \psi|^{2}+\frac{1}{4}|A|^{2}|\psi|^{2},
$$

which when inserted in (26) and recalling that the Riemannian measures of the two conformal metrics $\langle$,$\rangle and \langle,\rangle^{H}$ are related by

$$
d V^{H}=H^{n} d V,
$$


we finally obtain

$$
\begin{gathered}
\left\langle T_{\varphi}^{H}, h\right\rangle^{H} d V^{H}= \\
\left(\left(\Delta f+\frac{1}{2}|A|^{2} f+\overline{\operatorname{Ric}}(N, N) f\right)|\psi|^{2}+2\left(|\nabla \psi|^{2}-|\not \psi|^{2}\right) f\right) d V .
\end{gathered}
$$

Integration by parts of this formula, for $\psi$ belonging to the $m$-dimensional space $H^{\frac{n-1}{2}} E_{k}\left(\not D^{H}\right)$, shows that the quadratic form $Q_{h}$ (henceforth denoted by $Q_{f}$ since it depends only on $f$ ) takes the form

$$
\begin{gathered}
Q_{f}(\psi)= \\
-\frac{1}{2} \int_{M}\left(\Delta|\psi|^{2}+\frac{1}{2}|A|^{2}|\psi|^{2}+\overline{\operatorname{Ric}}(N, N)|\psi|^{2}+2|\nabla \psi|^{2}-2|\not \nabla|^{2}\right) f d V,
\end{gathered}
$$

and controls the derivatives $\left(d \mathcal{F}_{k}^{(i)} / d t\right)(0), i=1, \ldots, m$ of the $m$ analytic branches of the eigenvalue $\lambda_{k}(\not D)$ of $\not D^{H}(t)$ at $t=0$. Note that the Laplacian of the squared length of an eigenspinor is easily computable. In fact, from the compatibility condition (4), we immediately have

$$
\Delta|\psi|^{2}=2|\nabla \psi|^{2}+2\left\langle\text { trace } \nabla^{2} \psi, \psi\right\rangle .
$$

But the rough Laplacian trace $\nabla^{2}$ acting on sections of the spin bundle $\$ M$ satisfies the celebrated Schrödinger-Lichnerowicz formula

$$
\operatorname{trace} \nabla^{2}=\frac{1}{4} R-\not D^{2}
$$

where $R$ is the scalar curvature of $M$. This and (23) imply

$$
\Delta|\psi|^{2}=2|\nabla \psi|^{2}+\frac{1}{2}\left(R-n^{2} H^{2}\right)|\psi|^{2}
$$

Finally, the Gauß equation relating the curvature tensor of the ambient space $\bar{M}$ and that of the hypersurface $M$ reads

$$
R=\bar{R}-2 \overline{\operatorname{Ric}}(N, N)+n^{2} H^{2}-|A|^{2},
$$

where $\bar{R}$ is the scalar curvature of the ambient manifold $\bar{M}$. Hence, the following equality for functions on $M$ :

$$
\Delta|\psi|^{2}=2|\not \psi|^{2}+\left(\frac{1}{2} \bar{R}-\overline{\operatorname{Ric}}(N, N)-\frac{1}{2}|A|^{2}\right)|\psi|^{2} .
$$

With the above facts in mind, we are now ready to give the following result which allows the characterization of immersed hypersurfaces for which the eigenvalue $\frac{n}{2}$ of $\not D^{H}$ is a critical point of the functional $\mathcal{F}_{k}$.

Proposition 9. Let $M$ be a compact orientable hypersurface immersed into a Riemannian spin manifold $\bar{M}$ and assume that the mean curvature $H$ of $M$ is positive. Moreover, suppose that $\frac{n}{2}=\lambda_{k}\left(\not D^{H}\right)$ belongs to the spectrum of the Dirac operator, corresponding to the conformal metric $\langle,\rangle^{H}$ and to the induced spin structure, with multiplicity $m$. 
Consider a normal variation $\iota(t)$ of the immersion $M$ with variational field given by $f N$, where $f$ is a smooth function on $M$, and let $\mathcal{F}_{k}(t)$ be the corresponding variation of the $k$-th eigenvalue of $\not^{H}$. Then, the values of the derivatives of $\mathcal{F}_{k}$ lie among the $m$ eigenvalues of the quadratic form

$$
\psi \in H^{\frac{n-1}{2}} E_{k}\left(\not D^{H}\right) \longmapsto Q_{f}(\psi)=-\int_{M}\left(|\nabla \psi|^{2}+\frac{1}{4} \bar{R}|\psi|^{2}\right) f d V .
$$

If we assume that the scalar curvature $\bar{R}$ is non-negative, it is then obvious that the quadratic expression depending on the spinor $\psi$, inside the parentheses, is non-negative. Under this assumption, choosing $f$ as the constant function equal to 1 , that is, considering parallel variations of the hypersurface $M$, the quadratic form $Q_{1}$ is non-positive on the $m$ dimensional vector space on which it is defined. Then all its eigenvalues will be non-positive. This leads to the following necessary condition for the immersion to be critical with respect to the functional $\mathcal{F}_{k}=$ $\lambda_{k}\left(\not D^{H}\right)$.

Theorem 10. Let $M$ be an n-dimensional compact orientable hypersurface of a Riemannian spin manifold $(\bar{M},\langle\rangle$,$) . Suppose that the$ scalar curvature $\bar{R}$ of $\bar{M}$ is non-negative and that the mean curvature $H$ of $M$ is positive with respect to a suitable choice of the normal. If $\frac{n}{2}=\lambda_{k}\left(\not D^{H}\right)$ belongs to the spectrum of the Dirac operator $\not^{H}$ of the metric $H^{2}\langle$,$\rangle and it is critical for all the variations of the hypersur-$ face $M$, then $\bar{R} \equiv 0$ on $M$ and $\nabla \psi=0$ for any spinor field $\psi$ on $M$ satisfying

$$
\not D \psi=\frac{n}{2} H \psi
$$

Corollary 11. Let $M$ be an $n$-dimensional compact orientable hypersurface of a Riemannian spin manifold $(\bar{M},\langle\rangle$,$) whose mean curvature$ $H$ is positive. Moreover, suppose that there exists a non trivial parallel spinor field on $\bar{M}$. Then the first non-negative eigenvalue $\lambda_{1}\left(D^{H}\right)$ of the metric $H^{2}\langle$,$\rangle is at most \frac{n}{2}$. If the equality holds, then $\nabla \psi=0$ for any spinor field $\psi$ on $M$ such that $\not D \psi=\frac{n}{2} H \psi$.

Proof. To apply Theorem 10 it suffices to consider that $\bar{R}$ is identically zero because of the existence of a non trivial parallel spinor field on $\bar{M}$ (see, for example, [BFGK, Fr]) and recall that, in the presence of such a parallel spinor field, Proposition 8 with the fact that $\lambda_{1}\left(\not D^{H}\right)=$ $\frac{n}{2}$ imply that the immersion attains the maximum for $\lambda_{1}\left(\not D^{H}\right)$, since small deformations of hypersurfaces preserve the positivity of the mean curvature.

q.e.d. 
Remark 6. Note that the necessary condition $\nabla \psi=0$ obtained in Corollary 11 does not mean necessarily that the eigenspinor $\psi$ is the restriction to $M$ of a parallel spinor field on $\bar{M}$. The reason is that $\psi$ is defined only on the hypersurface $M$. Furthermore, it is interesting to point out that, according to (8), $\nabla \psi=0$ is equivalent to

$$
\nabla_{X} \psi=-\frac{1}{2} \not(A X) \psi, \quad \forall X \in \Gamma(T M) .
$$

A spinor field $\psi$ satisfying such an overdetermined system is usually called a generalized Killing spinor.

Proof of Theorem 3. In order to prove Theorem 3 in Section 1, assume that the ambient space is the Euclidean space $\mathbb{R}^{n+1}$ and take a non-trivial parallel spinor field $\Psi$ on it. From Proposition 7, we know that the restriction of $\Psi$ to $M$ satifies $\not D \Psi=\frac{n}{2} H \Psi$. Define $\psi:=\gamma(H x+N) \Psi$, where $x$ is the identity (or the position vector) map on $\mathbb{R}^{n+1}$. Then, for each $X \in \Gamma(T M)$ we have

$$
\nabla_{X} \psi=\gamma(H X-A X) \Psi
$$

since $H$ is constant. Then, if $\left\{e_{1}, \ldots, e_{n}\right\}$ is a local orthonormal basis tangent to $M$, we have

$$
\sum_{i=1}^{n} \gamma\left(e_{i}\right) \nabla_{e_{i}} \psi=\sum_{i=1}^{n} \gamma\left(e_{i}\right) \gamma\left(H e_{i}-A e_{i}\right) \Psi=-\operatorname{trace}(H \operatorname{Id}-A) \Psi=0 .
$$

Hence, all the spinor fields of the form $\gamma(H x+N) \Psi$, where $\Psi$ is an Euclidean parallel spinor field, are also eigenspinors corresponding to the eigenvalue $\frac{n}{2} H$. Suppose that the hypersurface $M$ is not umbilical. In this case, the map $\Psi \mapsto \gamma(H x+N) \Psi$ is injective, since if $\gamma(H x+$ $N) \Psi=0$ for some $\Psi$, then $H x+N=0$, considering that $\Psi$ has a non trivial constant length. But the condition $H x+N=0$ means that $M$ is a hypersphere of radius $\frac{1}{H}$ centered at the origin. Moreover, this last space cannot contain any restriction to $M$ of a Euclidean parallel spinor field. In fact, if $\gamma(H x+N) \Psi=\Phi$ for suitable Euclidean parallel spinor fields $\Psi$ and $\Phi$, then we would get $\nabla(\gamma(H x+N) \Psi)=0$ and so, using (27), we would deduce that $A=H \mathrm{Id}$, that is, the hypersurface $M$ would be totally umbilical. Now, it is well-known that the space of parallel spinor fields on the Euclidean space $\mathbb{R}^{n+1}$ is exactly $2^{\left[\frac{n+1}{2}\right]}$ dimensional. Then, from the reasonings above, the multiplicity of the eigenvalue $\frac{n}{2} H$ is at least

$$
\operatorname{mult} \not D\left(\frac{n}{2} H\right) \geq 2 \cdot 2^{\left[\frac{n+1}{2}\right]}=2^{\left[\frac{n+1}{2}\right]+1},
$$


provided that the hypersurface $M$ is not umbilical. This completes the proof of Theorem 3 when $n$ is even. In fact, in this case, from c) and d) in Proposition 6, we have that $\pm \frac{n}{2} H$ are both eigenvalues of $D_{M}$ and that

$$
\text { mult }_{D_{M}}\left( \pm \frac{n}{2} H\right)=\operatorname{mult}_{\not D}\left(\frac{n}{2} H\right) .
$$

As a consequence, since $n$ is even,

$$
\text { mult }_{D_{M}}\left( \pm \frac{n}{2} H\right) \geq 2^{\left[\frac{n+1}{2}\right]+1}=2^{\left[\frac{n}{2}\right]+1},
$$

if the hypersurface $M$ is not umbilical.

When $n$ is odd, for the above arguments, we consider parallel spinor fields $\Psi$ on the even-dimensional Euclidean space $\mathbb{R}^{n+1}$ with positive (resp. negative) chirality and of spinor fields of the form $\gamma(H x+N) \Phi$, where $\Phi$ is an Euclidean parallel spinor field with negative (resp. positive) chirality. It is well-known that the spaces of parallel spinor fields with positive and negative chirality in the even-dimensional Euclidean space $\mathbb{R}^{n+1}$ have both dimension exactly $2^{\left[\frac{n}{2}\right]}$. Now, taking into account b) in Proposition 6, we have

$$
\text { mult }_{D_{\mid \not M_{ \pm}}}\left(\frac{n}{2} H\right) \geq 2 \cdot 2^{\left[\frac{n}{2}\right]}=2^{\left[\frac{n}{2}\right]+1}
$$

provided that the hypersurface $M$ is not umbilical. Using e) in the same Proposition 6, we obtain that both $\pm \frac{n}{2} H$ are eigenvalues of $D_{M}$ and that

$$
\text { mult }{ }_{D_{M}}\left( \pm \frac{n}{2} H\right) \geq 2^{\left[\frac{n}{2}\right]+1}
$$

if the hypersurface $M$ is not umbilical.

q.e.d.

Proof of Theorem 4. For this, suppose that $M$ is a compact orientable hypersurface of the Euclidean space $\mathbb{R}^{n+1}$ satisfying the equality in the Bär inequality (1). Equality implies that the restriction $\psi \in \Gamma(\mathbb{S} M)$ of each parallel spinor field of $\mathbb{R}^{n+1}$, used as a test spinor in the Rayleigh quotient for $\not^{2}$, must be an eigenspinor associated with the eigenvalue $\lambda_{1}(\not D)^{2}$. So, applying the operator $\not D$ to $\not D \psi=\frac{n}{2} H \psi$, it follows

$$
\not D^{2} \psi=\frac{n}{2} \not(\not \nabla H) \psi+\frac{n^{2}}{4} H^{2} \psi=\lambda_{1}(\not D)^{2} \psi
$$

Since $\psi$ and $\psi_{(}(\not H) \psi$ are orthogonal, we conclude that $H$ is constant and $\lambda_{1}(\not D)=\frac{n}{2} H$. From Remark 4, Proposition 8 and Corollary 11, we conclude that the eigenspace associated with the eigenvalue $\frac{n}{2} H$ of the Dirac operator $\not D$, consists only of the restrictions of Euclidean parallel fields. Then mult $\not \supset\left(\frac{n}{2} H\right)=2^{\left[\frac{n+1}{2}\right]}$ and we apply (28) in the proof of Theorem 3 to finish the proof.

q.e.d. 
Remark 7. Note that, in spite of the fact that most of the results in this paper hold for hypersurfaces of Riemannian spin manifolds admitting a non trivial parallel spinor, Theorems 3 and 4 are stated only for hypersurfaces immersed in the Euclidean space. A careful reading of the proofs shows that the use of the position vector field $x$ defined on $\mathbb{R}^{n+1}$ is crucial. In other words, a key ingredient is the existence of a vector field $x \in \Gamma\left(T \mathbb{R}^{n+1}\right)$ such that $\nabla_{X} x=X$ for all $X \in \Gamma\left(T \mathbb{R}^{n+1}\right)$. But the existence of such a vector field is a characteristic feature of the cone over an arbitrary $n$-dimensional Riemannian manifold $P$, that is the $(n+1)$-dimensional Riemannian manifold $\bar{M}$ defined as $\bar{M}=] 0,+\infty[\times P$ endowed with the following warped product metric:

$$
\left.\langle,\rangle_{\bar{M}}=d r^{2}+r^{2}\langle,\rangle_{P}, \quad \forall r \in\right] 0,+\infty[.
$$

In fact (see [O'N, Chapter 7, p. 204]), the vector field $x$ given by

$$
x=r \frac{\partial}{\partial r} \in \Gamma(T \bar{M})
$$

plays the role of the vector position field in the Euclidean space, that is $\nabla_{X} x=X$ for all $X \in \Gamma(T \bar{M})$. Note that for $P=\mathbb{S}^{n}$ we have $\bar{M}=\mathbb{R}^{n+1}$, and this is the only case where the singularity at the vertex of the cone can be removed. Now, it was a nice observation by Bär (see [Bä1], [Wa2, Section 5] and [Ga]) that the cone $\bar{M}$ is a Riemannian spin manifold with a non trivial parallel spinor provided that the factor $P$ is taken to be a Riemannian spin manifold endowed with a non trivial real Killing spinor. Assuming for simplicity that $P$ is 1-connected and besides the basic case $P=\mathbb{S}^{n}$, the manifold $P$ (see [Bä1]) could be either an Einstein-Sasaki Riemannian manifold or a 7-dimensional $\operatorname{Spin}(7)$-manifold or a 6-dimensional nearly-Kähler manifold of constant type 1 . For any such a choice of $P$, the corresponding cone $\bar{M}$ is a Riemannian spin manifold endowed with a non trivial parallel spinor and a position vector field. Hence, up to slight adjustments on the hypothesis and the conclusion, the arguments in the proof of Theorems 3 and 4 extend to compact orientable hypersurfaces $M$ immersed in such a cone $\bar{M}$. More precisely, if either the mean curvature $H$ of $M$ is constant and mult $\not D\left(\frac{n}{2} H\right)<2 \cdot \operatorname{dim} \mathcal{P}(\bar{M})$, where $\mathcal{P}(\bar{M})$ is the space of parallel spinor fields on the cone $\bar{M}$, or equality is attained in the Bär inequality, then $M$ is totally umbilical. Now, one can apply [Mo, Lemma 4], where the second author classified all the umbilical compact hypersurfaces with constant mean curvature in a warped product of type $I \times{ }_{f} P$, where $f$ is a positive smooth function defined on the open interval $I$, whose Ricci tensor satisfies a "certain condition". In particular, such a condition is automatically satisfied when the warped 
product is Ricci-flat. Since the existence of a parallel spinor implies that the cone $\bar{M}$ is Ricci-flat, we may apply this classification result in [Mo] for $I=\mathbb{R}^{+}$and $f(r)=r^{2}$. The conclusion is that the umbilical hypersurface $M$ has necessarily to be a slice $\{r\} \times P$ for some $r \in] 0,+\infty[$, unless in the trivial case $P=\mathbb{S}^{n}$ where the ambient cone $\bar{M}$ is $\mathbb{R}^{n+1}$ (in this case, there are obviously other umbilical hypersurfaces, namely, hyperspheres not centered at the origin).

\section{REFERENCES}

[AF] I. Agricola, T. Friedrich, Upper bounds for the first eigenvalue of the Dirac operator on surfaces, Journ. Geom. Phys., 301 (1999), 1-22.

[Al] A.D. Alexandrov, A characteristic property of spheres, Ann. Mat. Pura Appl., 58 (1962), 303-315.

[Am1] B. Ammann, The Willmore conjecture for immersed tori with small curvature integral, Manuscripta Math., 101 (2000), 1-22.

[Am2] B. Ammann, Ambient Dirac eigenvalue estimates and the Willmore functional, in "Dirac Operators: Yesterday and Today", International Press, 2005. Editors: Bourguignon, Branson, Chamseddine, Hijazi, Stanton, 221-228.

[Bä1] C. Bär, Real Killing spinors and holonomy, Commun. Math. Phys., 154 (1993), 509-521.

[Bä2] C. Bär, Metrics with harmonic spinors, Geom. and Funct. Anal., 6 (1996), 899-942.

[Bä3] C. Bär, Extrinsic bounds of the Dirac operator, Ann. Glob. Anal. Geom., 16 (1998), 573-596.

[Bm] H. Baum, An upper bound for the first eigenvalue of the Dirac operator on compact spin manifolds, Math. Z., 206 (1991) 409-422.

[BFGK] H. Baum, T. Friedrich, R. Grünewald, I. Kath, Twistor and Killing Spinors on Riemannian Manifolds, Seminarbericht 108, HumboldtUniversität zu Berlin, 1990.

[BG] J.P. Bourguignon, P. Gauduchon, Spineurs, opérateurs de Dirac et variations de métriques, Commun. Math. Phys., 144 (1992), 581-599.

[BlW] D. Bleecker, J. Weiner, Extrinsic bound of $\lambda_{1}$ of $\Delta$ on a compact manifold, Comment. Math. Helv., 51 (1976), 601-609.

[BHMM] J.P. Bourguignon, O. Hijazi, J.L. Milhorat, A. Moroianu, A spinorial approach to Riemannian and conformal geometry, Monograph (In Preparation).

[Bu] J. Bureš, Dirac operators on hypersurfaces, Comment. Math. Univ. Carolin. 34 (1993), no. 2, 313-322.

[BFLPP] F. Burstall, D. Ferus, K. Leschke, F. Pedit, U. Pinkall, Conformal geometry of surfaces in $S^{4}$ and quaternions, Lect. Notes in Math., 1772 2002.

[Ch] B.Y. Chen, On the total curvature of immersed manifolds IV: Spectrum and total mean curvature, Bull. Math. Acad. Sinica, 7 (1979), 301-311.

[FLPP] D. Ferus, K. Leschke, F. Pedit, U. Pinkall, Quaternionic holomorphic geometry: Plücker formula, Dirac eigenvalue estimates and energy estimates of harmonic 2-tori, Invent. Math., 146 (2001), 507-593. 
[Fr] T. Friedrich, Dirac Operators in Riemannian Geometry, A.M.S. Graduate Studies in Math., vol. 25, 2000.

[FK] T. Friedrich, E. C. Kim, The Einstein-Dirac equation on Riemannian spin manifolds, J. Geom. Phys. 33 (2000), 128-172.

[Ga] S. Gallot, Équations différentielles caractéristiques de la sphère, Ann. Scient. Éc. Norm. Sup. 12 (1979), 235-267.

[Gi] N. Ginoux, The Dirac Spectrum, Lecture Notes in Mathematics, 1976 (2009).

[GWW] C. Gordon, D.L. Webb, S. Wolpert, One cannot hear the shape of a drum, Bull. Amer. Math. Soc., 27 (1992), 134-138.

[Hi1] O. Hijazi, A conformal lower bound for the smallest eigenvalue of the Dirac operator and Killing spinors, Commun. Math. Phys., 104 (1986), 151-162.

[Hi2] O. Hijazi, Lower bounds for the eigenvalues of the Dirac operator, J. Geom. Phys., 16 (1995), 27-38.

[HM] O. Hijazi, S. Montiel, Extrinsic Killing spinors, Math. Z., 244 (2003), $337-347$.

[HMR1] O. Hijazi, S. Montiel, A. Roldán, Eigenvalue boundary problems for the Dirac operator, Commun. Math. Phys., 231 (2002), 375-390.

[HMR2] O. Hijazi, S. Montiel, A. Roldán, Dirac operators on hypersurfaces of manifolds with negative scalar curvature, Ann. Global Anal. Geom., 23 (2003), 247-264.

[HMZ1] O. Hijazi, S. Montiel, X. Zhang, Dirac operator on embedded hypersurfaces, Math. Res. Lett, 8 (2001), 195-208.

[HMZ2] O. Hijazi, S. Montiel, X. Zhang, Eigenvalues of the Dirac operator on manifolds with boundary, Commun. Math. Phys., 221 (2001), 255-265.

[HMZ3] O. Hijazi, S. Montiel, X. Zhang, Conformal lower bounds for the Dirac operator of embedded hypersurfaces, Asian J. Math., 6 (2002), 23-36.

[Hit] N. Hitchin, Harmonic spinors, Adv. in Math., 14 (1974), 1-55.

[Ho] H. Hopf, Differential geometry in the large, Lecture Notes in Math., 1000, 1983.

[HTY] W.Y. Hsiang, Z.H. Teng, W.C. Yu, New examples of constant mean curvature immersions of $(2 k-1)$-spheres into Euclidean $2 k$-space, Ann. Math., 117 (1983), 609-625.

$[\mathrm{K}] \quad$ M. Kac, Can one hear the shape of a drum? Amer. Math. Monthly, 73 (1966), 1-23.

[Ka1] N. Kapouleas, Compact constant mean curvature surfaces in Euclidean three-space, J. Diff. Geom., 33 (1991), 683-715.

[Ka2] N. Kapouleas, Constant mean curvature surfaces constructed by fusing Wente tori, Invent. Math., 119 (1995), 443-518.

[LM] H.B. Lawson, M.L. Michelsohn, Spin Geometry, Princeton Math. Series, vol. 38, Princeton University Press, 1989.

[Mo] S. Montiel, Unicity of constant mean curvature hypersurfaces in some Riemannian manifolds, Indiana Univ. Math. J., 48 (1999), 711-748.

[MR] S. Montiel, A. Ros, Minimal immersions of surfaces by the first eigenfunctions and conformal area, Invent. Math., 83 (1986), 153-166.

[O] K. Ogiue, Some open problems in differential geometry, Proc. Symp. Pure Math., vol. XXVII (1973), 407-411. 
[O’N] B. O'Neill, Semi-Riemannian Geometry, Academic Press, 1983.

[Re] R.C. Reilly, On the first eigenvalue of the Laplacian for compact submanifolds of Euclidean space, Comment. Math. Helv., 52 (1977), 525-533.

[T] I.A. Taimanov, Dirac operator and conformal invariants of tori in 3-space, Tr. Mat. Inst. Steklova, 244 (2004), 249-280.

[Tr] A. Trautman, The Dirac operator on hypersurfaces, Acta Phys. Plon., B 26 (1995), 1283-1310.

[Wa1] M.Y. Wang, Parallel spinors and parallel forms, Ann. Global Anal. Geom., 7 (1989), 59-68.

[Wa2] M.Y. Wang, On non-simply connected manifolds with non-trivial parallel spinors, Ann. Global Anal. Geom., 13 (1995), 31-42.

[We] H.C. Wente, Counterexample to a conjecture of H. Hopf, Pacific J. Math., 121 (1986), 193-243.

[Y] S.T. Yau, Problem section, Ann. Math. Studies, 102 (1982), 669-706.

(Hijazi) Institut Élie Cartan, Université Henri Poincaré, Nancy I, B.P. 239, 54506 Vandeuvre-Lès-Nancy Cedex, France

E-mail address: hijazi@iecn.u-nancy.fr

(Montiel) Departamento de Geometría y Topología, Universidad de Granada, 18071 Granada, Spain

E-mail address: smontiel@ugr.es 